NAL PROPOSAL NO. 171

Scientific Spokesman:

J. J. Lord

Department of Physics

University of Washington

Seattle, Washington 98105

FTS/Off-Net: $206-583-0111$

$543-2777$

\title{
PROPOSED EMULSION EXPERIMENT \\ SEARCH FOR SHORT LIVED PARTICLES AT HIGH ENERGIES
}

Peter Kotzer

J. J. Lord

University of Washington

Apri1 19, 1972 
Department of Physics

University of washington

Seattle, Wa. 98105

\section{PROPOSED EMULS ION EXPERIMENT}

SEARCH FOR SHORT LIVED PARTICLES AT HIGH ENERGIES

To: Dr. Donald Getz, Secretary

Program Advisory Committee

National Accelerator Laboratory

Batavia, IlIinois 60510

From: Peter Kotzer and J. J. Lord

Department of Physics

University of Washington

Seattle, Wa. 98105

ApriI 19, 1972

Scientific spokesman: J. J. Lord, Prof. of Physics

Telephone: $\frac{206}{\text { or }} \quad \begin{array}{r}543-2777 \\ 542770\end{array}$
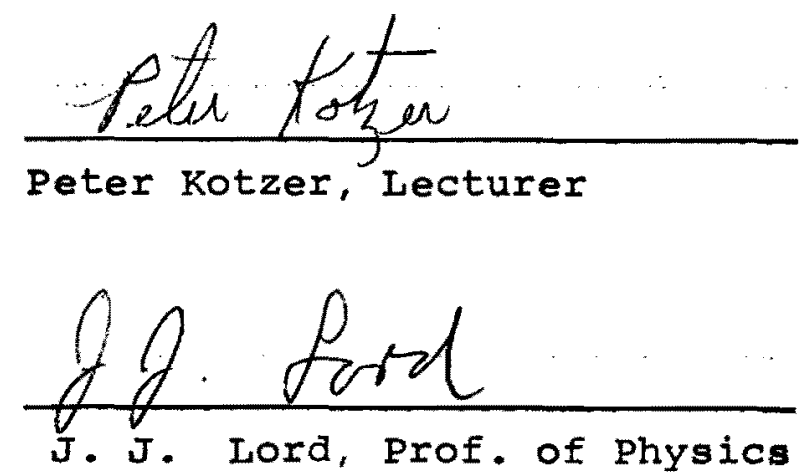


\section{ABSTRACT}

It is proposed to conduct an experiment at the National Accelerator Laboratory concerned with the search for new particles having lifetimes between $10^{-17}$ and $10^{-12}$ second. The experiment will be conducted with an emulsion chamber arrangement and will explore for particles with lifetimes too short to be observed directly with other instruments. Two sidelines of the experiment will be concerned with an improved measurement of the lifetime of the neutral pion and the search for gamma ray bundles arising from higher mass resonances which cannot decay by pion emission.

\section{INTRODUCTION}

The present-day knowledge of particle production in hadronic collisions is in a somewhat embryonic stage. At least several hundred boson and baryon resonances are considered by theoreticians making calculations with thermodynamic models for particle production. While the properties of resonances are most important, there is reason to feel that current observational techniques and analysis have not yielded significant results commensurate with the effort during the past year or two. Often experiments are found to be in rough agreement with semi-empirical theoretical models, leaving one with the impression that little of fundamental importance has been learned.

While it is important to introduce techniques which go beyond those in use at the present time, it is perhaps more easily said than done. However, it may be in this way that it 
is possible to find some special features of particle properties or production which will disclose something of the fundamental nature of the dynamics of particle physics.

The present proposal is concerned with an effort to search for new particles produced by 200-500 Gev protons at the National Accelerator Laboratory, Batavia. The search will be concerned with particles produced in collisions in the nuclear emulsion and having lifetimes in the range of $10^{-17}-10^{-12}$ second. The nuclear emulsion is the only instrument which allows direct measurements to be made in this range of lifetimes.

It is presumptuous, of course, to assume that the present proposed experiment will yield information of a fundamental nature. None-the-less, the experiment is of a modest nature and, in the light of recent cosmic ray observations, it could yield new information of a basic nature.

\section{PROPOSED EXPERIMENT}

A possible unusual particle was reported by Niu, Mikumo, and Maeda in the Proceedings of the 12 th International Conference on Cosmic Rays (Vol. 3 page 1262, Aug. 16-25, 1971). The event was produced in emulsion by a cosmic ray interaction of energy near 10,000 Gev. One emitted particle (described in the paper included in Appendix I of this proposal) traveled a distance of $1.38 \mathrm{~cm}$ and then appeared to decay into a charged particle and a $\pi^{\circ}$ meson. The gamma ray energies from the $\pi^{\circ}$ were determined and the unknown particle found to have a lifetime of about $10^{-14} \mathrm{sec}$. and a mass of about $2 \mathrm{Gev} / \mathrm{c}^{2}$. Since the particle had an energy of several Tev, the lifetime and mass values are not too strongly dependent upon the assumed 
mode of decay as shown in Appendix I. In the same event another particle traveled $4.88 \mathrm{~cm}$ before decay but its mass and lifetime couldn't be determined.

While one observation doesn't yield a meanlife, Niu's particle seems to have a lifetime quite different from that for known particles. If, on the other hand, the two unstable particles come from the decay of another very short lived particle, its mass would be about 5-10 Gev as estimated from the angles given in Fig. 6C of Niu's paper.

At first glance it looks as if Niu's particle (or particles) should have been observed in experiments at CERN, Brookhaven, or Serpukhov. Since there is a good deal of uncertainty in the particle mass estimates, it could be below the threshhold for production at all accelerators except the ISR at CERN. In the ISR its lifetime may make its detection rather difficult. Another question which may be raised is why it hasn't been observed before in cosmic ray experiments. Niu's use of a plastic-emulsion sandwich stack rather than one of pure emulsion produced a very much slower build-up of electromagnetic cascades. This enabled him to follow tracks out to distances of at least $10 \mathrm{~cm}$ before they were obscured by the tracks due to cascades. Niu's experiment is included as an illustration of the importance of conducting a search for new particles in the lifetime range from $10^{-17}-10^{-12}$ second. Basically however, the justification for such a search must be that it is highly important, at the beginning stages of an accelerator, to carry out a few exploratory experiments of a general nature. Hence, it is presently proposed to carry out an experiment similar to that of 
Niu's and search for new particles arising from nuclear interactions produced by 200-500 Gev particles.

Another experiment which would be a sideline of this proposal would be concerned with the lifetime of the neutral pion. Direct measurements of the $\pi^{\circ}$ lifetime have been made many years ago by observing the decay distance of the particle through the decay mode $\pi^{0}+\mathrm{e}^{+}+\mathrm{e}^{-}+\gamma$. The observations were made in emulsion and the distance measured from the production point of the $\pi^{\circ}$ to the origin of the decay electron-positron pair. The average distances were about 0.3 microns compared with the emulsion grain size of about 0.9 microns (after processing). Emulsion measurements gave a mean life of about $1.4 \pm 0.4 \times 10^{-16}$ second while counter experiments through the Primakoff effect gave about $0.7^{ \pm} 0.06 \times 10^{-16}$ second. There is need for a better value of the mean life of the neutral pion.

Neutral pions produced by particles at the NAL energies will have time dilatations about a factor of 10 greater than in the previous experiments. The consequent decay distance in the emulsion would be about 10 times larger which would allow the lifetime to be determined to a precision of about 88 rather than the 308 as given above. The scanning methods used in the experiment to search for new particles would automatically locate the above described neutral pion decay vertices. There would be little additional effort required to make the measurement of the decay distance and to estimate the energy of the pair. EXPERIMENTAL ARRANGEMENT

Several different types of detectors will be used in the search for new particles produced by the high energy hadronic 
interactions at NAL. The main features of the design will be to have a detector which is capable of allowing observations to be made on the decay of short lived particles.

In collisions at 200-500 Gev, speculation can only be made regarding the mode of production and energies of new particles. From particle spectra at Serpukov and the scaling ideas of Bali, Brown, Peccei, and Pignotti, it can be assumed that there will be a good deal of secondary particles emitted at energies of 15 to $20 \mathrm{Gev}$. Without speculation on the masses or modes of production, assume a time dilation of $\gamma=10$ for the new particles. In this case lifetimes of $10^{-17}$ to $10^{-12}$ second will lead to decay paths from $3 \times 10^{-5} \mathrm{~mm}$ to $3 \mathrm{~mm}$. While the best resolution of the emulsion is about $3 \times 10^{-4} \mathrm{~mm}$, the lower lifetime limit would still allow the detection of lighter particles. For example, the neutral pion having a time dilation value of $\gamma=500$ would have a decay distance of $15 \times 10^{-3} \mathrm{~mm}$, about 50 times greater than the resolution of the emulsion. Unstable particles such as those found by Niu et al would have a mean decay length of the order of 0.1 to $0.5 \mathrm{~mm}$ at NAL energies. This is based on extrapolations from the cosmic ray experiments wherein the time dilation factor would be reduced by a factor of about 100. In addition, for decay distances greater than about $1 \mathrm{~mm}$, observations could be made much more effectively with bubble chambers.

Niu et al found their events out of a sample of about a dozen interactions which had undergone careful examination. At NAL energies we plan to examine about 5000 interactions for evidence of decays or pair production within $1 \mathrm{~mm}$ of the point 
of interaction. Although one emulsion plate would provide sufficient statistics for the experiment, a large stack will be needed to study the nature of the tracks after a decay. As shown in Fig. 1, a stack $7 \mathrm{~cm} \times 15 \mathrm{~cm} \times 3 \mathrm{~cm}$ will provide the main detector for the experiment. There would be 50 pellicles in the stack each 600 microns in thickness and made of Ilford G-5 emulsion.

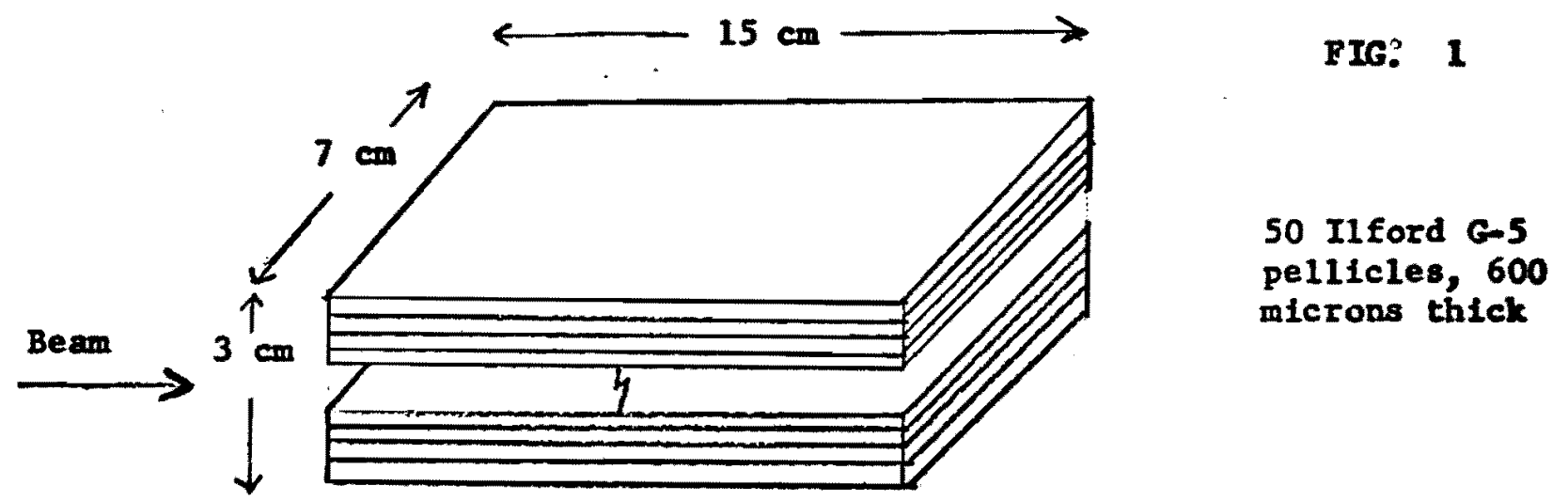

It is proposed to expose the stack to 90,000 particles $/ \mathrm{cm}^{2}$ of the highest energy available at NAL. This intensity is about the maximum that will allow individual tracks to be followed with sufficient precision to locate evidence for a decay. Naturally, many test exposures would be made to be sure of the proper flux.

Several smaller stacks will be made up to study the high energy events with a view of detecting gamma rays. The arrangement, shown in Fig. 2, is made up of a sandwich of emulsions and lead plates. It is held together in a frame made of two brass pins which allow alignment of the plates with a precision of about 5 microns. A larger precision sandwich stack of this type has been described by Gibbs and Lord (Acta Physica Academiae 29 Supp1. 3, 113, 1970). The proposed arrangement shown in 
Fig. 2 will be employed to search for gamma rays from the high energy nuclear interactions or the decay products of unstable particles.

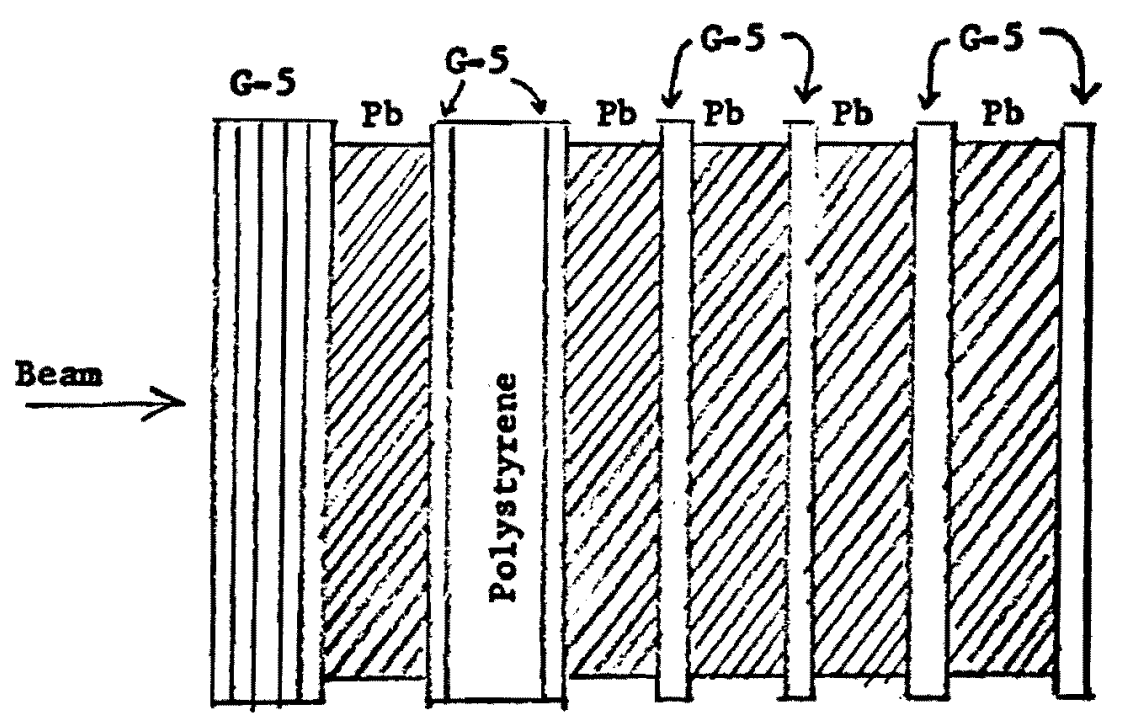

FIG: 2

All emulsions are Ilford G-5

As shown in Fig. 2 there will be a small producing region consisting of 5 pellicles, 600 microns in thickness, located on the beam side of the detector. After that there will be a $1 \mathrm{~mm}$ thick $\mathrm{Pb}$ plate ( $1 / 5$ radiation length in thickness) followed by an 800 micron thick polystyrene plate with 300 microns of emulsion on either side. The latter double coated emulsion allows accurate angle measurements to be made for single tracks and electron pairs. An additional sandwich of $4 \mathrm{~Pb}$ plates and emulsions provides about 1 radiation length of material between the high energy event and the detector plate. This arrangement is similar to the gamma ray detection system employed by Niu et al. It will allow the detection of gamma rays originating from the decay of the unstable particles being sought in this experiment. Naturally, in this ca'se, the gamma rays will be of very much lower energy than found in the cosmic ray experiment. However, the alignment arrangement of the stack will allow accurate 
angular measurements of individual electron-positron pairs for correlation with decay events.

The producing layer as shown in Fig. 2 is quite thin and a flux of 10,000 particles per $\mathrm{cm}^{2}$ through the five pellicles will give about one interaction per $\mathrm{mm}^{2}$ of the cross sectional area. This density of events is sufficient to allow good correlation of the gamma rays with events.

While many experiments with counters and bubble chambers will search for new particles at the National Accelerator Laboratory, they will be restricted to those having lifetimes larger than $10^{-12} \mathrm{sec}$. or the very short lifetimes of the resonances. The present experiment is designed to search in a region not accessible to counter or bubble chambers, ie. $\tau=10^{-17}$ $10^{-12}$ second. It is also a search wherein there is cosmic ray evidence for a particle of lifetime $10^{-14}$ second and having a mass less than perhaps $3 \mathrm{Gev}$.

The proposed experiment is simple and can be carried out with only one or two pulses of particles from the accelerator.

A second sideline experiment which is possible with the arrangement of Fig. 2 will be the search for ganma ray bundles arising from the decay of higher mass resonances which cannot decay by pion emission. The regge trajectories for meson and baryon families have at most only three or four members. The meson trajectories vary linearly with the square of the rest mass while the baryon trajectories are linear with respect to the mass. At higher $J$ values $(5$ or 6 ) the difference in mass 
may be less than the rest mass of the pion. In this case the only decay mode might be through the emission of a gamma ray with $\Delta J=1$. If the $J$ is quite large the meson might decay it stepwise into four or five gamma rays. A the NAL energies a small bundle of gamma rays would be emitted.

In the proposed experiment the emulsion sandwich detector will have $1 \mathrm{~mm}$ lead plates for the purpose of detecting gamma rays. This feature of the detector would give a finite chance to locate and estimate the energy of a gamma ray bundle. While gamma ray bundles will be looked for in the scanning, the emulsion sandwich detector is not too satisfactory an instrument in the search for low energy gamma rays. The main effort in the proposed experiment will remain, as noted before, the search for new particles as described above.

\section{ACCELERATOR ARRANGEMENTS REQUIRED}

While only two emulsion stacks are needed for this experiment, it would be well to prepare a few additional ones in order to obtain the optimum exposures. A summary of the proposed exposures follows:

\begin{tabular}{|c|c|c|c|}
\hline stack & Approx. size & $\begin{array}{l}\text { Type of particle } \\
\text { and energy }\end{array}$ & Total exposure \\
\hline$\# 1$ & $2 " \times 3 " \times 6 "$ & $2 \cdot 1-500 \mathrm{Gev}$ & 53,003 per $\mathrm{cm}^{2}$ \\
\hline$\# 2$ & $2 " \times 3^{M} \times 6 "$ & $200-500 \mathrm{GeV}$ & 90,000 per $\mathrm{cm}^{2}$ \\
\hline$\# 3$ & $2 " \times 3 " x 6 "$ & $200-500 \mathrm{GeV}$ & 120,000 per $\mathrm{cm}^{2}$ \\
\hline$\# 4$ & $5 " \times 5 " \times 4 "$ & $200-500 \mathrm{Gev}$ & 10,000 per $\mathrm{cm}^{2}$ \\
\hline$\# 5$ & $5 " \times 5 " x 4 "$ & $200-500 \mathrm{GeV}$ & 30,000 per $\mathrm{cm}^{2}$ \\
\hline$\# 6$ & $2 " \times 3 " x 6 "$ & neutrons $200-500 \mathrm{Gev}$ & 500,000 per $\mathrm{cm}^{2}$ \\
\hline$\# 7$ & $2 " \times 3 " \times 6 "$ & neutrons $200-500 \mathrm{Gev}$ & 800,000 per $\mathrm{cm}^{2}$ \\
\hline
\end{tabular}


(from: Proceedings of 12 th Int. Conf. on Cosmic Rays, Vol. 3, page 1262,

Observation of High Energy Interactions

Aug. 1971) at Aeroplane Altitute

K. Niu and E. Mikumo

Institute for Nuclear Study, University of Tokyo

Y. Maeda

Yokohama National University

An analysis is presented on a nuclear interaction observed by emulsion chambers with producing layers exposed at an aeroplane altitude. Among secondary particles produced in a high energy jet shower, a possible decay in flight of a particle of unknown type was found.

1. Introduction. In order to investigate super high energy jet showers in full detail combining the informations about secondary charged particles at or near an origin of jet shower as well as $Y$ ray component, a new type of emulsion chamber with producing layer was developped, and exposed to cosmic rays. The measurement and analysis on jet showers observed are now going on, and hore the report is limited only ahout an event already analysed.

2. Design of the chamber. The emulsion chamiers for this experiment consisted of three parts as shown in Fig. 1. These are: upper chamber as a detector of $\gamma$ rays coming from the upper atmosphere; middle chamber with material producing jet shower, which is also used as an analyser of charged component from jet shower; and lower chaniher as an analyser, as well as a detector of $\gamma$ rays produced hy the jet shower in the middle chamber.

To minimize the development of cascade showers and occurrence of secondary jet showers initiated

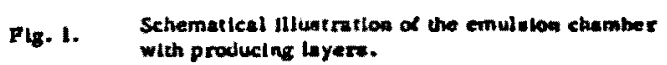
by secondary particles in the producer layer, and to observe a crossectional view of jet showers each 1 man along the shower axis, we adopted, as a producer, a pile of nuclear emulsion plates with a $50 \mu \mathrm{m}$ coating on both sides of meta-acryl base with a thickness of $800 \mu \mathrm{m}$.

A typical design of the chamber is shom in Fig. 2. The middle chamber is a pile of 49 layers of nuclear emulsion plates with $X$-ray films and 5 lead plates with thickness of $1 \mathrm{~nm}$. The thickness of this pile is $7.3 \mathrm{~cm}$ and this corresponds to 0.1 nuclear interaction mean free path and 0.3 (Emulsion plates) +1.0 (Lead plates) cascade unit. The total size of this chamber is $20 \mathrm{~cm} \times 25 \mathrm{~cm} \times$ $19 \mathrm{~cm}$, and the weight is about $50 \mathrm{~kg}$ including the packing case. 
3. Fxposure and scanning. Fxposure of this type of cinulsion chamber in the upper atmosphcre, ahout $260 \mathrm{gr} / \mathrm{cm}^{2}$, was performed using a Jet Cargo Neroplane of Japan Air lines from August to December 1969. In tota1, 12 chambers were exposed with an effective exposure of $400 \sim 600$ hours.

The scanning for jet showers was made through the following scanning program. $\Lambda t$ first, there is selection of those multi $y$ rays events in the lower chamher whose primary particle come through upper chamber leaving no shore, and next following back shower tracks up into the producing layers and detection of the origin of jet shower.

\section{Event "6B-23"}

a) General description of the event. One of jet shower observed in these chamber, Event "6B-23", was fortunately produced in the emulsion of lower surfase of plate $\mathrm{No} .38$ in the middle of the producer layer. Therefore, type of this jet shower was observed as $(19+70) \mathrm{n}$. Schematical view of this jet shower is shown in Fig. 3 .

Analysing on cascade showers of this event in the lower chamber we get 23 cascade cores. Frission angles of charged particles, were estimated using target diagramns on each emulsion surface in the producer layer. Following down these particles into the lower chamber, * we found that 4 of the 23 cascade showers belong to secondary interactions induced by charged secondary particles.

Target diagramn of cascade cores at plate No.7, 6.63 cm from the origin of jet shower is shown in Fig. 4, with estimated energy of each cascade shower.

Pairs of $y$ rays coupled with line in this figure are nosible $\pi^{\circ}$ mesons whose production hights are coincide with the origin of the jet shower within the error of 10 8. From this analysis our method of energy determination of $Y$ rays was confirmed.

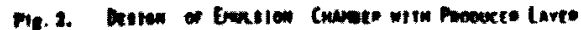

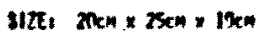
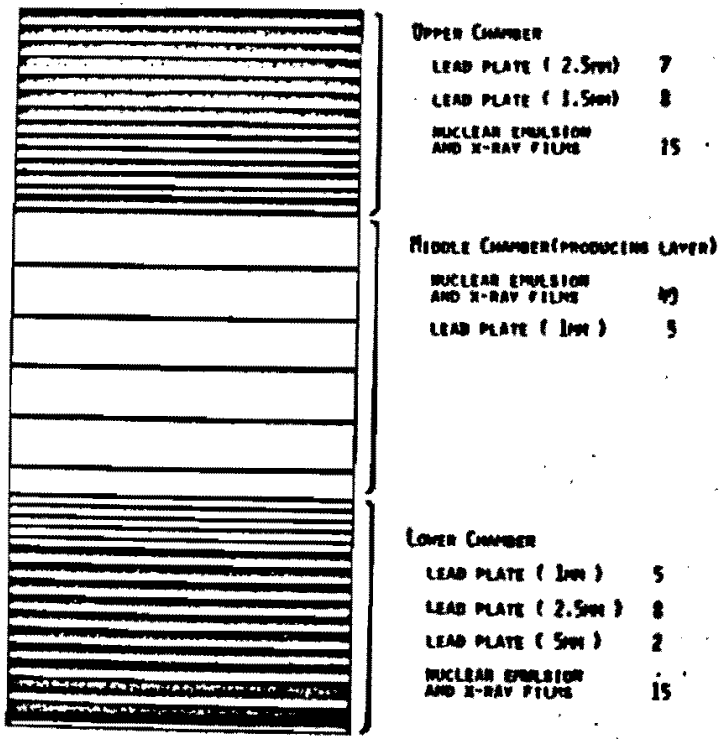

Lant commen (xes nare ( In) 4 e mart $(2 . \mathrm{sm})$ ? (we mate $(\sin )$ axcun ematen is
P18 * 3

EVENT 6B-23

$(19+70) n$

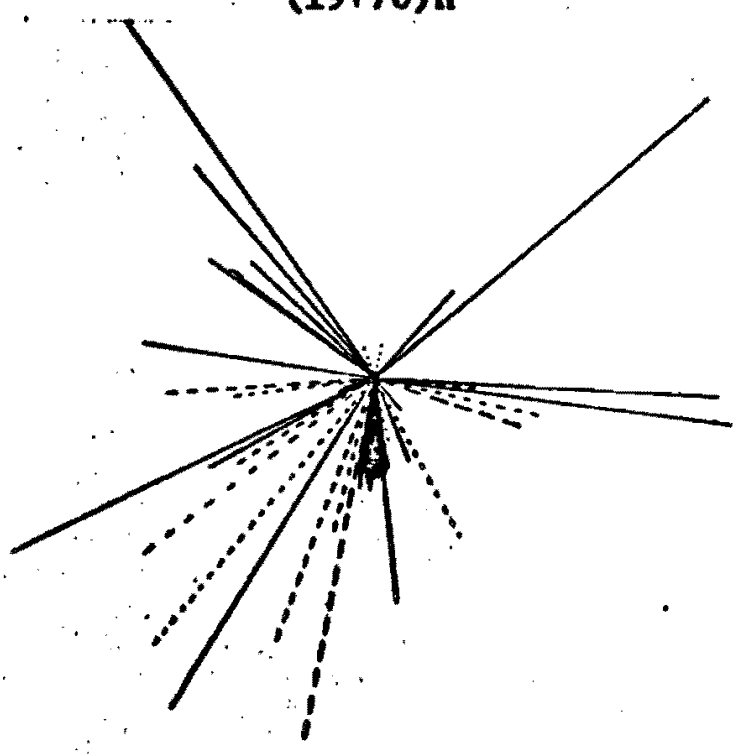


In Fig. 5 is shown the relation of encrgy to $1 g \cdot \operatorname{tg} \theta$ for $\gamma$ rays with Ig.tgo distribution of charged scconchry particles. Though the jet shower "6B$23^{\prime \prime}$ is produced by a conplicated collision of noutral priman particle with $\Lambda \mathrm{g}$ or $\mathrm{Br}$ nuclei in the emulsion, the angular distribution of charged particles shows two fire ball like structure. Comparing anguler distribution of $Y$ rays with that of charged particles, it is seen that we observed neary $2 / 3$ of $\gamma$ rays of forward fire ball. From this, threshold energy to detect $\gamma$ rays in this case turns out to be about $40 \mathrm{Gev}$.

b) Central part of the event. The most impressive feature of this jet shower is that $2 \gamma$ rays whose energy are nearly one order higher than others are emitted very closely in the center region of shower. Relative distance hetiveen those $Y$ rays is 3.4 $\mathrm{im}_{\mathrm{m}}$ at the plate No. 10. Because of a very small distance between them, independent energy detenmination for each $Y$ ray could not be possible. We estimated total energy of these $\gamma$ rays as $3.2 \pm 0.4$ Tev. Compareing size of hoth cores, cnergy ratio of these two $Y$ rays is estimated as $\sim 2: 1$. Coupling these two $\gamma$ rays into a $\pi^{~}$ meson, the estimated production hight is $3.8 \pm 0.5 \mathrm{~cm}$ ahove the plate No. 10 , and it is smaller than the hight of origin of primary jet shower, $5.14 \mathrm{~cm}$.

There cinitted 4 charged particles very near to these $\gamma$ rays. We drew piesise target diagrann of tracks of these 4 particles $A, B, C$, and $D$ at each cmulsion surface in the producer layer, and then reconstructed three dimensional view of tracks of these particles and $\pi^{\circ}$ meson. $X, Y$, and $Z$ projection of these tracks are shown in Fig. $6 a, b$ and $c$. We adopted $z$ axis parallel to the emitted direction of track $B$.

As you can see from $X$ and $Y$ projections, track $B$ and $C$ have knees at $i .38$ on 
and 4.88 an respectively from the origin of jet shower and the angle of direction clinnge of tracks are $1.07 \times 10^{-3}$ radian, and $1.50 \times 10^{-3}$ radian respectively.

looking at the 7 projection, you can casily recognize that the two cascade cores from $\pi^{\circ}$ meson appear in just the opposite direction of $Z$ projection of track $B^{\prime}$, and particles $B, B^{\prime}$ and $\pi^{0}$ meson satisfy the coplanarity condition. The emission angle of $\pi^{\circ}$ meson from the point of direction change of track $R$ is $1.96 \times 10^{-4}$ radian.

lie followed dom the track $B$ ' and $C^{\prime}$ to the bottom of lower chamber the thickness of which is 7 cascade unit, and found nothing assosiated with tracks $B^{\prime}$ and $C^{\prime}$ before their lcaving the chamber. Therefore, the track $B^{\prime}$ and $C^{\prime}$ might be produced by hadronic particles. Inspecting near arround the point of direction change of track $B$ and $C$, no track assosiated with these points was found.

c) A possible two bndy decay of $X$ particle. Basing on the features mentioned ahove, it is possible to attribute the phenomenon including track $B, B^{\prime}$ and $\pi^{\circ}$ meson to a two body decay of $X$ particle into a $\pi^{\circ}$ meson and a charged hadron.

Transverse momentum of $\pi^{0}$ meson to the initial diroction of track $B$ is estimated as $627 \mathrm{Mcv} / \mathrm{C}$ from its erission angle and obscrved energy. This value is also applied for that of. particle $B^{\prime}$ in the case of two body decay. From this we get the moinentum of particle $B^{\prime}$ as $0.59 \mathrm{Tev} / \mathrm{C}$.

Assuming $\pi^{ \pm}$meson or proton as a partner of $\pi^{\circ}$ meson, mass and life time of $X$ particle are got as shown in Table 1.
4

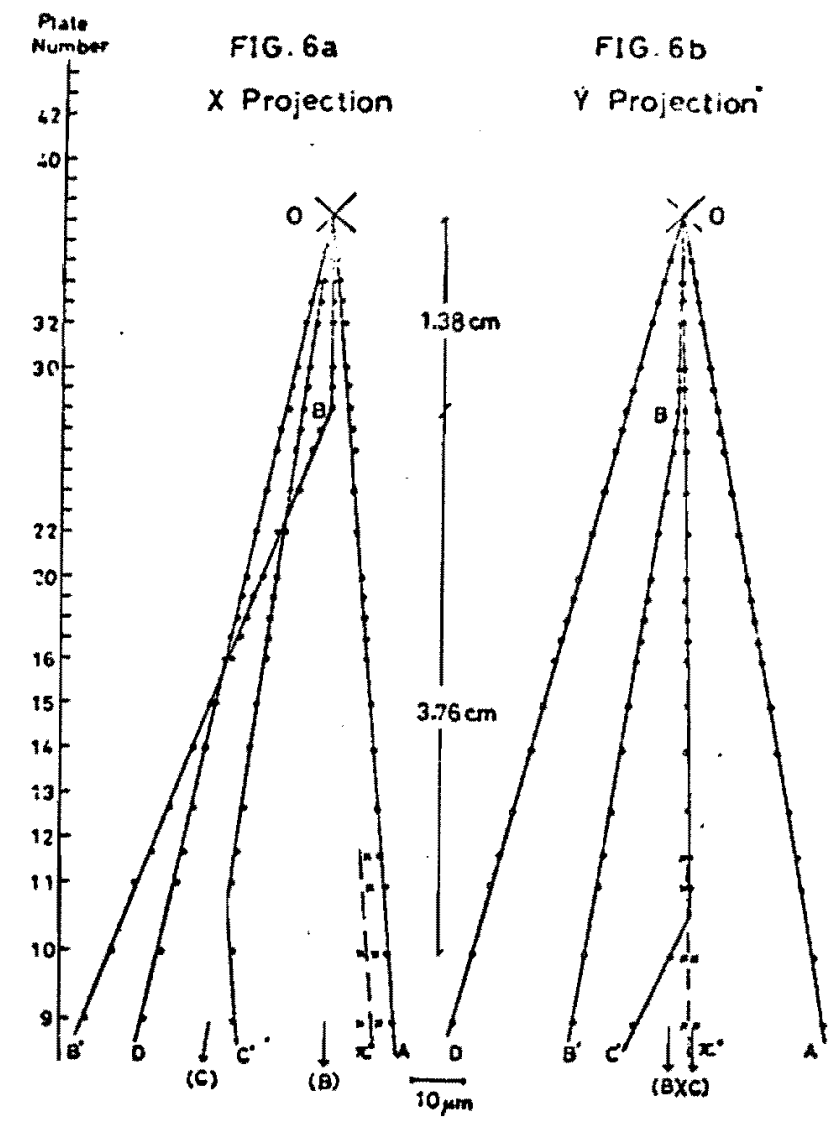

FIG.6c

$Z$ Projection

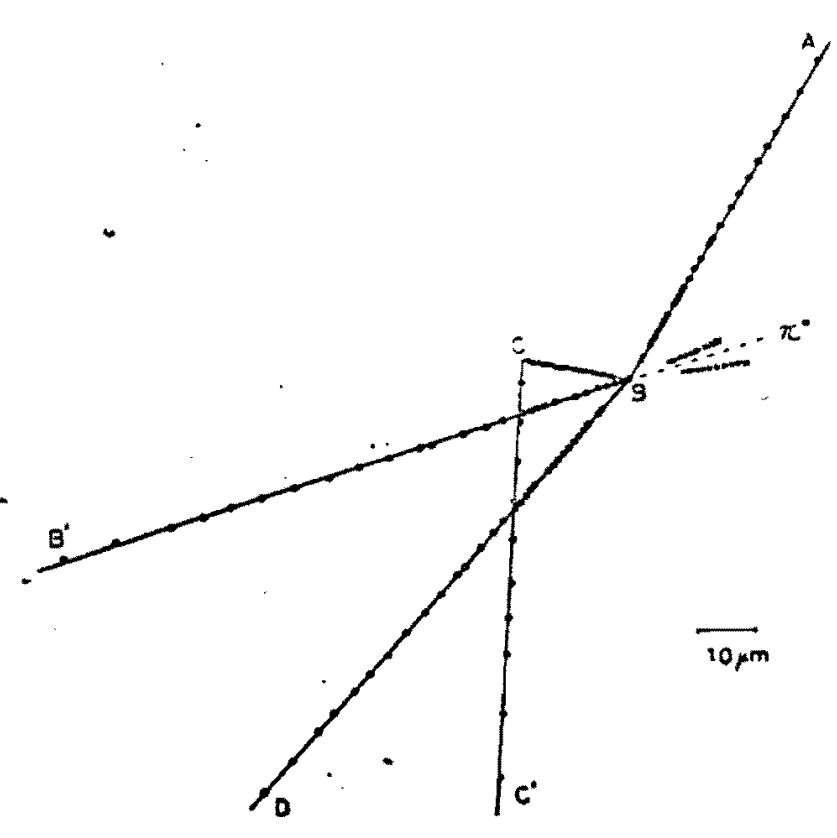


5. Discussions. As far as $\gamma$ rays concerned, there ohscrved sometimies "6B-23" like cvents in rmulsion Chamher Fiperiment on Mts. Norikura and Chacal tryai. 1) In Bristol Bombay Experiment ${ }^{2}$ ) with a halhon cxposed emulsion chamicr, there observed several such events moung 44 jet

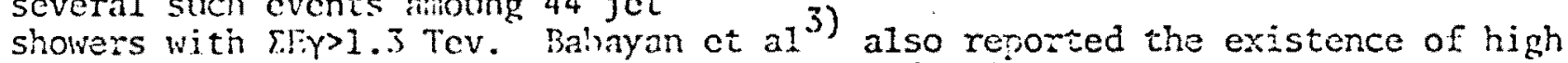
energy events in which appreciable fraction of primary energy is transferred to a single $\pi^{0}$ meson. Some of these events might have the same characteristics as our event "óB-23". Japan Brasii collaboration group of emulsion chamber called this type of events as "Torpecio", and they attributed this to a new state of matter with anisotropy. Combining the infomation about charged secondary particles as well as $t ! n$ Y ray component of jet shower, we were able to analyse this type of event more presisely and got above results.

The possibility of this type of event produced by secondary interaction or chance coincidence of elastic scattering of one of charged secondary particle and original $\pi^{0}$ meson so enitted as to satisfy coplanarity condition is very small as is show in Table 2.

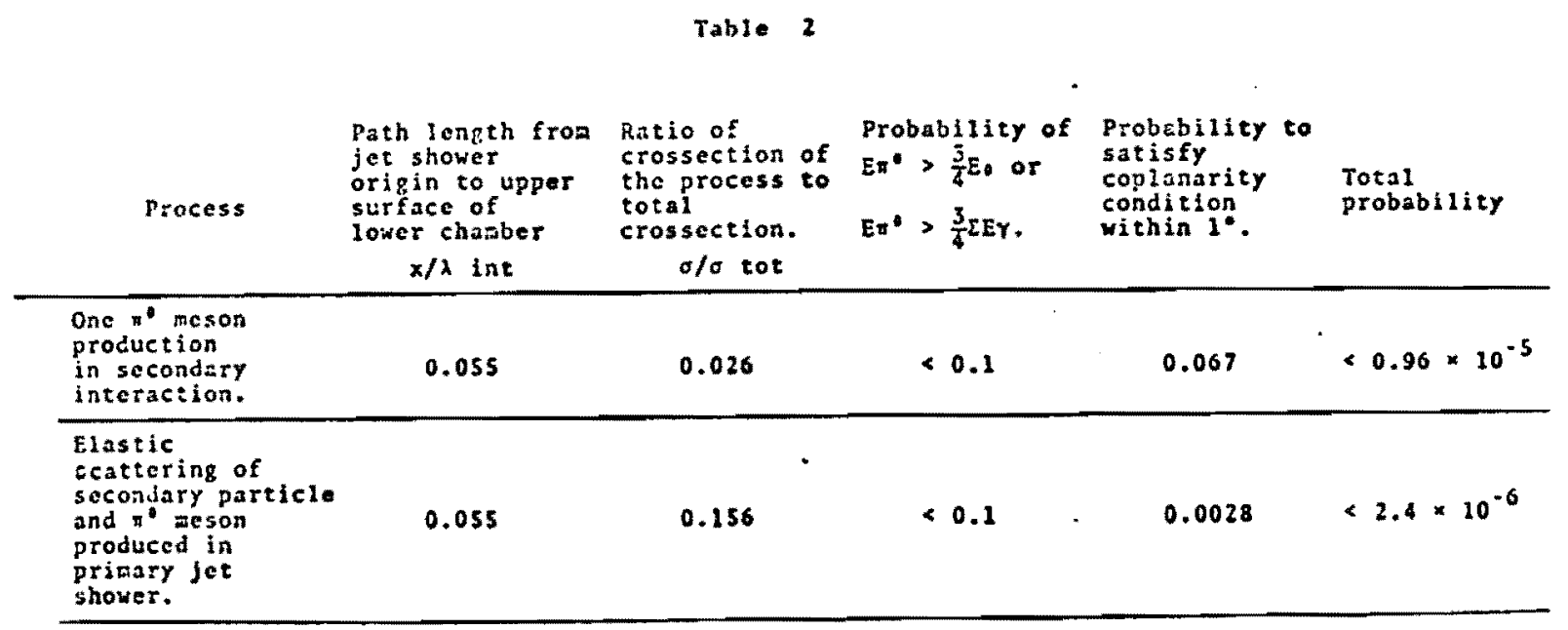

As for the charactoristics of $X$ particle the transverse monentun of $\pi^{0}$ meson, $627 \pm 90 \mathrm{Mev} / \mathrm{C}$, is muci higher than maximum momentum of decay products of existing strange particles. The lifo time of assuned $X$ particle is several times $10^{-14}$ seconds and this is extremely longer than those of resonance particles. Ticrefore, our $X$ particle could not be included neither in strange particle nor resonance particle.

If the dirccion change of track $C$ at $4.83 \mathrm{~cm}$ from jet snower origin is attributed to a decay of particle $C$ into a hadronic particle $C^{\prime}$ and a neutral hadrcnic particle, maybe ve are observing a pair production of new type of particles.

Being informed about this evont, s. Ogawa of al ${ }^{4)}$ reached a conclusion that 
our $X$ particle must be such a particle which has a new quantum number and is produced in pair in super high energy strong interactions. Attributing this to $p^{\prime}$ particic which is the fourth type of fundamental baryon corresponding to 4 leptons, and asstming hyperon like 2 body decay bia weak intaraction of this now particle with mass of 2.95 rev, they estimated mean life tine of this channel to be $3.2 \times 10^{-13} \mathrm{sec}$.

of course, it is also possible to attribute cie of the decay products of the $X$ particle which prodiced track $B^{\prime}$ or track $C^{\prime}$ to a $\mu$ meson. Then, there might be other possihilitics to interprete this type of event.

We are nnw continuing to search for new type of particles, and we have another possible example of pair producion of $X$ particles produced in supar high energy intcraction. One of charged secondary particle suffered direction change after ahout $1 \mathrm{~cm}$ flight, and another possibly decaycd into three charged hadronic particles after about 2 on flight. Full detail of this and other events wili be published elsewhere.

Finally Table 3 is a short sumary of reasons why thishof particle cannot be identified by other experinental method.

\begin{tabular}{|c|c|}
\hline Experimental method & $\begin{array}{l}\text { The reason why this type of particle } \\
\text { cannot be found. }\end{array}$ \\
\hline $\begin{array}{l}\text { Experiments in high } \\
\text { cnergy accelerator } \\
\text { region. }\end{array}$ & $\begin{array}{l}\text { The Lorentz factor and then the flighting } \\
\text { path of produced new particle, if it is } \\
\text { produced, is too smali to idetify. } \\
\text { in case of pair production, incident } \\
\text { energy is not sufficient because of } \\
\text { bigher threshold of production. }\end{array}$ \\
\hline $\begin{array}{l}\text { limulsinn chambar } \\
\text { experiment in cosmic } \\
\text { ray region. }\end{array}$ & $\begin{array}{l}\text { Observation is limitted mainly in r rays } \\
\text { and information about charged secondary } \\
\text { particles at or near the origin of jet } \\
\text { shower is omitced. }\end{array}$ \\
\hline $\begin{array}{l}\text { Emulsion stack } \\
\text { experiment in cosmic } \\
\text { ray region. }\end{array}$ & $\begin{array}{l}\text { One cascade unit corresponds to } 3 \mathrm{~cm} \\
\text { in emulsion stack and many rays initiate } \\
\text { cascade showers earior than let them } \\
\text { identify this type of particle. }\end{array}$ \\
\hline $\begin{array}{l}\text { other track detecter } \\
\text { or counter experiments } \\
\text { in cosmic ray region. }\end{array}$ & $\begin{array}{l}\text { Observation is limitted mainly in } \\
\text { charged secondary particles at more than } \\
\text { several cm down from jet shower origin } \\
\text { and observation of individual y ray. } \\
\text { is hardly possible. }\end{array}$ \\
\hline
\end{tabular}

Acknonledgoments. The authors would like.to express their gratitude to memhers of Japan $\wedge$ ir Lines, especially to Mrtilarada, for making successful exposures at aeroplane altitudes. Thanks are also due to Dr. T. Sueyoshi of Fuji Film Company for making emulsion plates coated on both sides of thin meta-acryl bases.

\section{References}

1) Japancse and Brasilian Fmulsion Chamber Group; 1971

Surpl. Prog. Tneor. Phys. No.46.

2) Nalhotra, P. K. et al; 1965. Nov. Cim. 40A, 404.

3) Babayan, Kh. P. et al; 1964. J. Expil. Theoret. Prys (USSR) 47, 379.

4) Gzawa, S. et al; 1971. to be published in Prog. Theor. Phys. 